\title{
A Case of Episodic Ataxia Type-2 with a Novel Gene Mutation and Com- plex Phenotype
}

\author{
Rossi FH ${ }^{1 *}$, Rossi EM¹, Gonzalez $\mathrm{E}^{1}$, Franco $\mathrm{MC}^{2}$ and Estevez $\mathrm{AG}^{2}$
}

${ }^{1}$ Department of Neurology, Veteran Health Care System, Orlando, P.O. Box: 32827, Florida, USA

${ }^{2}$ Burnett School of Biomedical Sciences, College of Medicine, University of Central Florida, Orlando, P.O. Box: 32827, Florida, USA

\begin{abstract}
We report the case of 66-year-old male with episodic ataxia type 2 carrying a novel CACNA1A gene mutation affected with a new and heterogeneous phenotype. The patient presented with intermittent episodes of ataxia, dysarthria, and vertigo since childhood. The patient also had atypical features of perioral numbness, right facial drooling, and bilateral upper limb action tremor. The cerebellar ataxia episodes significantly increased in frequency with age, but entirely remitted with acetazolamide treatment.
\end{abstract}

\section{Keywords: Cerebellar; Episodic; Atrophy; Ataxia; CACNA1A}

\section{Introduction}

Episodic ataxia type 2 (EA2) is a familial autonomic dominant disorder that present with paroxysmal cerebellar dysfunction and ataxia. EA2 is caused by mutations of the calcium channel CACNA1A gene mapped to chromosome 19p. The variability in EA2 phenotypes is commonly associated with different nonsense or missense mutations in the CACNA1A [1]. We report a man with EA2 caused by novel single base insertion in the CACNA1A gene associated with a complex neurological phenotype.

\section{Case Report}

66 years old white male presented paroxysmal episodes of ataxia, dysarthria, and vertigo since age 12. Episodes lasted 15 minutes to four hours. Initially episodes occurred twice a year, but in the last few years presented three times daily. Episodes were associated with perioral numbness, mild right facial drooling, and bilateral upper limbs action tremor. Episodes were triggered by stress. In between attacks, the patient was asymptomatic. The patient denied history of diplopia or visual deficits, impairment of consciousness, headaches or migraines, hemiplegia, or speech disturbances. The patient mother, maternal uncle and nice, as well as several maternal cousins suffered from intermittent episodes of ataxia. The patient had a history of diabetes, diabetic polyneuropathy, nephrolithiasis, hypertension and hyperlipidemia. Neurological examination between attacks revealed a mild left gaze nystagmus, vibratory loss at his legs, reduced tandem gait, positive Romberg, but no cerebellar dysfunction. His carotid ultrasound and head MRI/MRA were normal, there was no evidence of cerebellar atrophy (Figures 1 and 2). Laboratory tests were unremarkable. Genetic blood testing for EA2 showed a novel heterozygous variant within Exon 20: c.3535dupC, p.Leu1179fs. This mutation consisted of a duplication of a cytosine within a stretch of seven cytosines at nucleotide positions 3529-3535 (for a total of eight cytosines). This resulted in the change of the leucine at amino acid position 1179 to a proline and a shift in the reading frame thereafter, leading to a termination codon 38 codons beyond this change (Figure 3). Genetic tests for episodic ataxia type 1 (EA1) and spinocerebellar ataxia type 6 (SCA6) CAG repeats were negative. Treatment with acetazolamide (ACTZ) 250mg BID made the patient attack-free for seven years.

\section{Discussion}

EA2 is a rare autosomal dominant disorder that manifests with intermittent episodes of ataxia, vertigo, nystagmus, and dysarthria.
Less often patients have diplopia, tinnitus, hemiplegia, dystonia, and migraines. The episodes have a frequency of a few attacks per year to daily occurrence and last from minutes to hours. In between episodes, patients are asymptomatic or display a progressive cerebellar ataxia and nystagmus EA2 starts during childhood or early adolescence. Episodes are often triggered by stress, fever, heat, carbohydrate-rich meals, coffee, alcohol, and exercise. EA2 is caused by mutations of the CACNA1A gene encoding the $\mathrm{P} / \mathrm{Q}$ subunit of a calcium channel complex. These mutations often predict a premature stop due to defect on the splice site or are nonsense mutations. EA2 responds to acetazolamide [1-3]. This man presented with EA2 caused by a novel single base insertion that predict the expression of a truncated CACNA1A protein. This mutation manifested as paroxysmal attacks of cerebellar dysfunction and unusual features of perioral numbness, mild right facial drooling, and bilateral upper limbs action, not previously reported.

EA2 phenotypically overlaps with other three genetic disorders: spino-cerebellar ataxia type 6 (SCA6), familial hemiplegic migraine type 1 (FHM1), and episodic ataxia type 1 (EA1). EA2, SCA6, and FHM1 are all allelic disorders of the same CACNA1A gene [3]. SCA6 presents with a late-onset slowly progressive pure cerebellar disorder ataxia [4], differentiating it from EA2. However, SCA6 may initially present with intermittent episodes or have only a paroxysmal nature of cerebellar dysfunction. SCA6 is caused by a CAG repeat expansion mutation in the exon 47 of the CACNA1A gene $[5,6]$. This patient tested negative for SCA6 CAG expansion.

FHM1 is a migraine subtype that present with the typical aura of hemiplegia and migraine headaches; patients may have a superimposed episodic or progressive cerebellar dysfunction. FHM1 is caused by a missense mutation of the CACNA1A gene [1,7]. In this case, the lack of hemiplegia and migraines makes unlikely the diagnosis of FHM1.

*Corresponding author: Fabian H. Rossi, Orlando VA Medical Center, Lake Nona VAMC, 13800 Veterans Way, Orlando, Florida, P.O. Box: 32827, Tel: 4077196245; Fax: 4076311977; E-mail: Fabian.Rossi@va.gov

Received September 15, 2015; Accepted October 17, 2015; Published October 20 , 2015

Citation: Rossi FH, Rossi EM, Gonzalez E, Franco MC, Estevez AG (2015)A Case of Episodic Ataxia Type-2 with a Novel Gene Mutation and Complex Phenotype. J Neurol Disord S1: 004. doi:10.4172/2329-6895.S1-004

Copyright: ( 2015 Rossi FH, et al. This is an open-access article distributed under the terms of the Creative Commons Attribution License, which permits unrestricted use, distribution, and reproduction in any medium, provided the original author and source are credited. 
Citation: Rossi FH, Rossi EM, Gonzalez E, Franco MC, Estevez AG (2015)A Case of Episodic Ataxia Type-2 with a Novel Gene Mutation and Complex Phenotype. J Neurol Disord S1: 004. doi:10.4172/2329-6895.S1-004

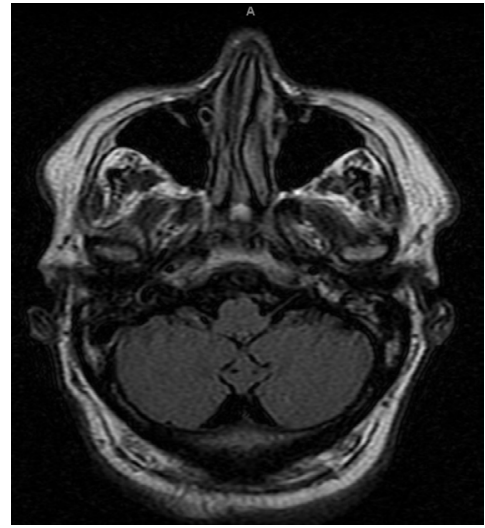

Figure 1: Axial FLAIR images showing a normal cerebellum.

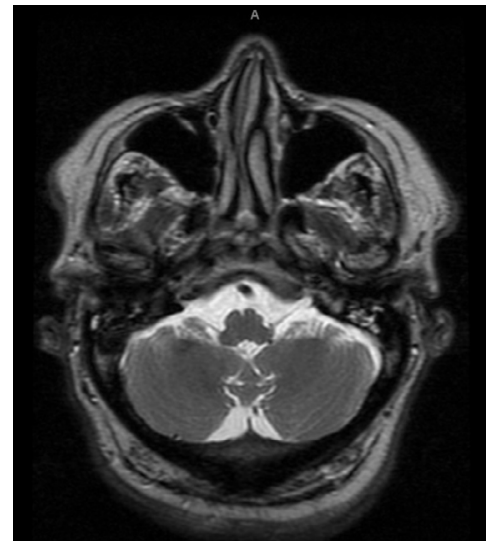

Figure 2: Axial T2-weighted images showing normal cerebellum.

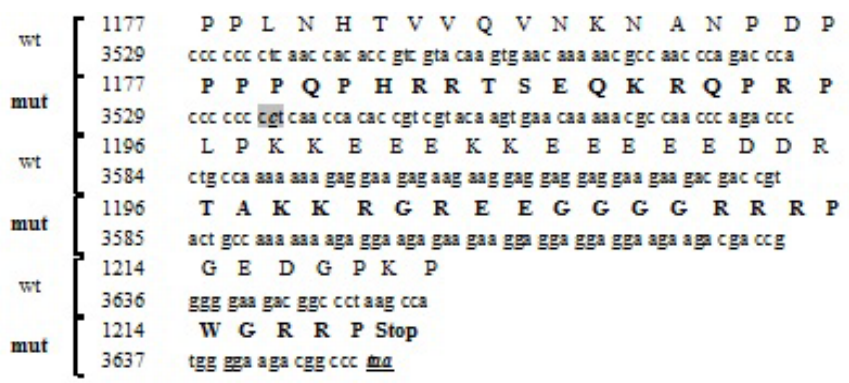

Figure 3: Genetic mutation within Exon 20: c.3535dupC, p.Leu1179fs.
Episodic ataxia type 1 (EA1) is a genetic disorder caused by a missense mutation in the potassium channel gene $\mathrm{KCNa}$ at chromosome 12q [3]. EA1 presents similar to EA2 with a paroxysmal cerebellar dysfunction, although attacks are brief (lasting less than 15 minutes), with a frequency up to 30 per day, in between episodes patients have facial and/or limbs myokymia, and the disease remit by the third decade of life. This patient did not share this presentation and tested negative for EA1.

We report a man with EA2 caused by a new CACNA1A gene mutation presenting with a complex neurological phenotype not reported before. Its recognition may facilitate the diagnosis and treatment of this genetic disorder.

\section{References}

1. http://www.ncbi.nlm.nih.gov/books/NBK1501

2. Strupp M, Zwergal A, Brandt T (2007) Episodic ataxia type 2. Neurotherapeutics 4: 267-273.

3. Jen JC, Graves TD, Hess EJ, Hanna MG, Griggs RC, et al. (2007) Primary episodic ataxias: diagnosis, pathogenesis and treatment. Brain 130: 24842493.

4. Schmitz-Hübsch T, Coudert M, Bauer P, Giunti P, Globas C, et al. (2008) Spinocerebellar ataxia types, 3 , and 6 : disease severity and nonataxia symptoms. Neurology 71: 982-989.

5. Jodice C, Mantuano E, Veneziano L, Trettel F, Sabbadini G, et al. (1997) Episodic ataxia type 2 (EA2) and spinocerebellar ataxia type 6 (SCA6) due to CAG repeat expansion in the CACNA1A gene on chromosome 19p. Hum Mol Genet 6: 1973-1978.

6. Mantuano E, Veneziano L, Jodice C, Frontali M (2003) Spinocerebellar ataxia type 6 and episodic ataxia type 2: differences and similarities between two allelic disorders. Cytogenet Genome Res 100: 147-153.

7. http://www.ncbi.nlm.nih.gov/books/NBK1388.
This article was originally published in a special issue, Management of Neurosurgical Complications handled by Editor(s). Dr. Athanasios K. Petridis, Department of Neurosurgery, University of Schleswig Holstein, Germany 